\title{
Monstrous and Generalized Moonshine and Permutation Orbifolds
}

\author{
Michael P. Tuite \\ School of Mathematics, Statistics and Applied Mathematics, \\ National University of Ireland, \\ Galway, Ireland.
}

June 14, 2021

\begin{abstract}
We consider the application of permutation orbifold constructions towards a new possible understanding of the genus zero property in Monstrous and Generalized Moonshine. We describe a theory of twisted Hecke operators in this setting and conjecture on the form of Generalized Moonshine replication formulas.
\end{abstract}

\section{Introduction}

The Conway and Norton Monstrous Moonshine Conjectures [CN], the construction of the Moonshine Module [FLM] as an orbifold vertex operator algebra and the completion of the proof of Monstrous Moonshine by Borcherds [B02] provided much of the motivation for the development of Vertex Operator Algebras (VOAs) e.g. [Bo1], FLM], [Ka], [MN]. Another highlight of VOA theory is Zhu's study of the modular properties of the partition function (and $n$-point functions) for generic classes of VOAs [Z]. Zhu's ideas were generalized to include orbifold VOAs [DLM] whose relevance to Monstrous Moonshine is emphasized in refs. [T1], [T2]. Norton's Generalized Moonshine Conjectures [N2] concerning centralizers of the Monster group has yet to be generally proven using either Borcherds' approach or orbifold partition function methods although some progress has recently been made in refs. $\mathrm{H}$ and [T3, [T1], [IT2] respectively.

In this note, we sketch a possible new approach to these areas based on permutation orbifold VOA constructions [DMVV], BDM]. In particular, we introduce a theory of twisted Hecke operators generalizing classical Hecke 
operators in number theory e.g. [Se]. We then discuss permutation orbifold constructions where the classical Hecke operators naturally appear and finite group and permutation orbifold constructions where the twisted Hecke operators appear. Using these ideas we formulate a conjecture on the nature of Generalized Moonshine replication formulas generalizing replication formulas for the classical $J$ function and McKay-Thompson series in Monstrous Moonshine. Detailed proofs will appear elsewhere [T4].

\section{Replication Formula for the $J$ Function}

We begin with a brief review of Faber polynomials, Hecke algebras and the replication formula for the classical $J$ function. Consider

$$
t(q)=q^{-1}+0+\sum_{k \geq 1} a(k) q^{k}
$$

the formal series in $q$. Define the Faber polynomial $P_{n}(x)$ for $t(q)$ to be the unique $n^{\text {th }}$ order polynomial with coefficients in $\mathbb{Z}[a(1), \ldots, a(n-1)]$ such that

$$
P_{n}(t(q))=q^{-n}+O(q)
$$

Thus $P_{1}(x)=x, P_{2}(x)=x^{2}-2 a(1), P_{3}(x)=x^{3}-3 a(1) x-3 a(2)$ etc. The Faber polynomials for $t$ satisfy the following generating relation e.g. [Cu, N1]

$$
\exp \left(-\sum_{n \geq 1} \frac{p^{n}}{n} P_{n}(x)\right)=p(t(p)-x),
$$

for formal parameter $p$.

Let $f(\tau)$ be a meromorphic function of $\tau \in \mathbb{H}$, the upper half complex plane. Then for integer $k \geq 1$ define a right modular group action on $f$ for $\gamma=\left(\begin{array}{ll}a & b \\ c & d\end{array}\right) \in \Gamma=S L(2, \mathbb{Z})$ as follows

$$
\left(\left.f\right|_{k} \gamma\right)(\tau)=(c \tau+d)^{-k} f(\gamma \tau)
$$

with $\gamma \tau=\frac{a \tau+b}{c \tau+d}$. Then $f$ is a modular form of (necessarily even) weight $k$ if $f$ is holomorphic in $\tau$ and

$$
\left.f\right|_{k} \gamma=f .
$$

Define the standard Hecke operators $T(n)$ for $n \geq 1$ with the following action on a modular form $f$ of weight $k$ Se]

$$
T(n) f(\tau)=\frac{1}{n} \sum_{a \geq 1, a d=n} a^{k} \sum_{0 \leq b<d} f\left(\frac{a \tau+b}{d}\right) .
$$


These satisfy the Hecke algebra

$$
\begin{aligned}
T(m n) & =T(m) T(n), \quad(m, n)=1, \\
T(p) T\left(p^{m}\right) & =T\left(p^{m+1}\right)+p^{k-1} T\left(p^{m-1}\right), \quad m \geq 1, \quad \text { prime } p .
\end{aligned}
$$

One finds

$$
\left.(T(n) f)\right|_{k} \gamma=T(n) f,
$$

i.e. $T(n) f$ is also a modular form of weight $k$.

The classical example is the Eisenstein series $G_{k}$ of even weight $k \geq 4$ (with $G_{k}(\tau)=0$ for odd $k$ ) [op.cit.]

$$
\begin{aligned}
G_{k}(\tau) & =\sum_{\substack{m, n \in \mathbb{Z} \\
(m, n) \neq(0,0)}} \frac{1}{(m \tau+n)^{k}} \\
& =2 \zeta(k)+2 \frac{(2 \pi i)^{k}}{(k-1) !} \sum_{n \geq 1} \sigma_{k-1}(n) q^{n},
\end{aligned}
$$

where $q=\exp (2 \pi i \tau)$ and $\sigma_{k}(n)=\sum_{d \mid n} d^{k}$. Furthermore, $G_{k}$ is an eigenfunction of $T(n)$ with eigenvalue determined by the coefficient of $q^{n}$ normalized to the coefficient of $q$ i.e.

$$
T(n) G_{k}=\sigma_{k-1}(n) G_{k} .
$$

Thus it follows from (5) that for $k$ odd

$$
\begin{aligned}
\sigma_{k}(m n) & =\sigma_{k}(m) \sigma_{k}(n), \quad(m, n)=1, \\
\sigma_{k}(p) \sigma_{k}\left(p^{m}\right) & =\sigma_{k}\left(p^{m+1}\right)+p^{k} \sigma_{k}\left(p^{m-1}\right), \quad m \geq 1, \text { prime } p .
\end{aligned}
$$

In fact, it is easy to check directly that (9) holds for all $k \in \mathbb{C}$.

The classical modular invariant function of weight 0 is given by

$$
\begin{aligned}
J(\tau) & =1728 \frac{G_{4}^{3}}{G_{4}^{3}-G_{6}^{2}}-744 \\
& =\sum_{k \in \mathbb{Z}} c(k) q^{k}=q^{-1}+0+196884 q+21493760 q^{2}+\ldots
\end{aligned}
$$

with standard normalization $c(-1)=1$ and $c(0)=0 . \quad J$ is a hauptmodul for the genus zero group $\Gamma$ and is thus a generator for the field of modular invariants. Thus

$$
\begin{aligned}
T(n) J(\tau) & =\sum_{a \geq 1, a \mid n} \frac{1}{a} \sum_{s \in \mathbb{Z}} c\left(\frac{n s}{a}\right) q^{a s} \\
& =\frac{1}{n} q^{-n}+O(q) .
\end{aligned}
$$


is a polynomial in $J$ which from (11) and (11) must be

$$
T(n) J(\tau)=\frac{1}{n} P_{n}(J(\tau)),
$$

where $P_{n}$ is the Faber polynomial corresponding to $J$. Eqn. (12) is called the replication formula for $J$.

Eqn. (10) also implies that

$$
\sum_{n \geq 1} p^{n} T(n) J(\tau)=\sum_{r \geq 1, s \in \mathbb{Z}} c(r s) \sum_{a \geq 1} \frac{1}{a} p^{a r} q^{a s}=-\sum_{r \geq 1, s \in \mathbb{Z}} c(r s) \log \left(1-p^{r} q^{s}\right) .
$$

Then (2) implies the famous $J$ function denominator formula [N1], Bo2]

$$
\begin{aligned}
\exp \left(-\sum_{n \geq 1} p^{n} T(n) J(\tau)\right) & =\prod_{r \geq 1, s \in \mathbb{Z}}\left(1-p^{r} q^{s}\right)^{c(r s)} \\
& =p(J(p)-J(q)) .
\end{aligned}
$$

This formula is one of the cornerstones of Borcherds' celebrated proof of the genus zero Moonshine property where (13) is a denominator formula for a particular generalized Kac-Moody algebra constructed from the Moonshine Module $V^{\natural}[\mathrm{Bo} 2]$.

\section{Twisted Hecke Operators and Eisenstein Se- ries}

The definitions of modular functions and Hecke operators above can be generalized to "twisted" versions as follows. We define a twisted modular form of integer weight $k$ to be a holomorphic (in $\tau$ ) function $f=f((\theta, \phi), \tau$ ) for $(\theta, \phi) \in U(1) \times U(1)$ such that

$$
\left.f\right|_{k} \gamma=f
$$

where

$$
\left(\left.f\right|_{k} \gamma\right)((\theta, \phi), \tau)=(c \tau+d)^{-k} f(\gamma(\theta, \phi), \gamma \tau)
$$

with left group action

$$
\gamma(\theta, \phi)=\left(\theta^{a} \phi^{b}, \theta^{c} \phi^{d}\right) .
$$

Clearly the case $(\theta, \phi)=(1,1)$ defines a standard modular form of weight $k$.

We can extend the definition of the Hecke operator $T(n)$ to twisted modular forms as follows:

$$
T(n) f((\theta, \phi), \tau)=\frac{1}{n} \sum_{a \geq 1, a d=n} a^{k} \sum_{0 \leq b<d} f\left(\left(\theta^{a} \phi^{b}, \phi^{d}\right), \frac{a \tau+b}{d}\right),
$$


which includes the standard definition in the case $(\theta, \phi)=(1,1)$. For $\phi=1$ and $\theta^{m}=1$ for integer $m$, this Hecke operator is essentially that which appears in Borcherds' proof [Bo2 and is discussed at length in ref. $F$.

We also define a homothety operator 1

$$
R(n) f((\theta, \phi), \tau)=f\left(\left(\theta^{n}, \phi^{n}\right), \tau\right) .
$$

These operators satisfy the Hecke algebra [T4]

$$
\begin{aligned}
R(m n) & =R(m) R(n) \\
R(m) T(n) & =T(n) R(m) \\
T(m n) & =T(m) T(n), \quad(m, n)=1, \\
T(p) T\left(p^{m}\right) & =T\left(p^{m+1}\right)+p^{k-1} T\left(p^{m-1}\right) R(p), \quad m \geq 1, \quad \text { prime } p,
\end{aligned}
$$

and one again finds

$$
\left.(T(n) f)\right|_{k} \gamma=T(n) f
$$

i.e. $T(n) f$ is also a twisted modular form of weight $k$.

A twisted Eisenstein series $\left.G_{k}((\theta, \phi), \tau)\right)$ of weight $k \geq 1$ can also be defined [DLM], [MTZ]. In particular, for $k \geq 4$ we define ${ }^{2}$

$$
G_{k}((\theta, \phi), \tau)=\sum_{\substack{m, n \in \mathbb{Z} \\(m, n) \neq(0,0)}} \frac{\theta^{m} \phi^{n}}{(m \tau+n)^{k}}
$$

for $\theta, \phi \in U(1)$ with $\left.G_{k}((1,1), \tau)\right)=G_{k}(\tau)$ [MTZ. $G_{k}((\theta, \phi), \tau)$ is not an eigenfunction of $T(n)$ in general. However, for prime $p$

$$
T(p) G_{k}((\theta, \phi), \tau)=p^{k-1} G_{k}((\theta, \phi), \tau)+R(p) G_{k}((\theta, \phi), \tau) .
$$

Hence if $(\theta, \phi)=\left(\theta^{p}, \phi^{p}\right)$ then $T(p) G_{k}((\theta, \phi), \tau)=\sigma_{k-1}(p) G_{k}((\theta, \phi), \tau)$.

\section{The Permutation Orbifold of a $C=24$ Holo- morphic Vertex Operator Algebra}

\subsection{The Orbifold of a Holomorphic VOA}

We now consider a Vertex Operator Algebra $V$ (VOA) of central charge 24 e.g. [FLM], [Ka], [MN]. We assume that $V$ is a Holomorphic VOA (HVOA)

\footnotetext{
${ }^{1} \mathrm{~A}$ similar operator is defined in the standard case $\mathrm{Se}$.

${ }^{2}$ The notation used here differs from that of op.cit.
} 
so that $V$ is the unique irreducible module for itself with modular invariant meromorphic partition function [Sch, [DM]

$$
\begin{aligned}
Z_{V}(\tau) & =\operatorname{Tr}_{V}\left(q^{L(0)-1}\right)=\sum_{k \geq-1} a(k) q^{k} \\
& =J(\tau)+a(0) .
\end{aligned}
$$

For example, the Moonshine Module $V^{\natural}$ is a HVOA with $Z_{V^{\natural}}(\tau)=J(\tau)$ whereas $Z_{V_{L}}(\tau)=J(\tau)+24$ for the Leech lattice HVOA $V_{L}$ [FLM].

Let $G$ be a finite subgroup of the automorphism group of $V$. Then for $g \in G$ define the orbifold trace function

$$
Z_{V}((g, 1), \tau)=\operatorname{Tr}_{V}\left(g q^{L(0)-1}\right)
$$

(so that $\left.Z_{V}((1,1), \tau)=Z_{V}(\tau)\right)$. For the Moonshine Module $V^{\natural}$

$$
T_{g}(\tau)=Z_{V^{\natural}}((g, 1), \tau),
$$

is the McKay-Thompson series for $g \in \mathbb{M}$, the Monster group of automorphisms of $V^{\natural}$.

Since $V$ is holomorphic, there is a unique twisted module $M_{h}$ for each $h \in G$ [DLM]. For $g \in C(h)$, the $h$ centralizer, $g$ induces a class of linear maps $\phi(g)$ on $M_{h}$ so that we may define a twisted orbifold trace function 3

$$
Z((g, h), q)=Z((g, h), \tau)=\operatorname{Tr}_{M_{h}}\left(\phi(g) q^{L(0)-1}\right),
$$

a meromorphic function for $\tau \in \mathbb{H}$ [op.cit.]. We define a right action of the modular group for $\gamma \in \Gamma$ as follow 4

$$
\left(\left.Z\right|_{0} \gamma\right)((g, h), \tau)=Z(\gamma(g, h), \gamma \tau)
$$

with

$$
\gamma(g, h)=\left(g^{a} h^{b}, g^{c} h^{d}\right) .
$$

The trace function enjoys the modular invariance property

$$
\left(\left.Z\right|_{0} \gamma\right)((g, h), \tau)=\epsilon_{\gamma}(g, h) Z((g, h), \tau) .
$$

for cocycle $\epsilon_{\gamma}(g, h) \in \mathbb{C}^{*}$ [op.cit.] generalizing earlier ideas of Zhu concerning trace functions $[\mathbf{Z}$. Specializing to the McKay-Thompson series (20), these results imply that $T_{g}(\tau)$ is a meromorphic function on $\mathbb{H}$ satisfying the modular invariance property (23) for $h=1$.

\footnotetext{
${ }^{3}$ denoted by $Z\left(h, g^{-1}, \tau\right)$ in ref. DLM,

${ }^{4}$ The 0 subscript denotes the modular weight of $Z((g, h), \tau)$.
} 
Let us consider orbifolds without a global phase anomaly i.e. where each $\phi(g)$ acting on $M_{h}$ can be chosen such that $\epsilon_{\gamma}(g, h)=1$ so that [Va]

$$
\left(\left.Z\right|_{0} \gamma\right)((g, h), \tau)=Z((g, h), \tau) .
$$

In particular, this condition implies that for $h$ of order $m$

$$
Z((1, h), \tau)=\sum_{k \in \mathbb{Z}} a\left((1, h), \frac{k}{m}\right) q^{k / m},
$$

for some integers $a\left((1, h), \frac{k}{m}\right) \geq 0$. We next define the $G$-orbifold partition function by

$$
\begin{aligned}
Z^{G-\mathrm{orb}}(\tau) & =\frac{1}{|G|} \sum_{\substack{g, h \in G \\
g h=h g}} Z((g, h), \tau) \\
& =\sum_{[h] \in G} \frac{1}{|C(h)|} \sum_{g \in C_{G}(h)} Z((g, h), \tau),
\end{aligned}
$$

for centralizer $C(h)=\{h g=g h \mid g \in G\}$ and where $[h]$ denotes a conjugacy class of $G$. Clearly $Z^{G-o r b}(\tau)$ is also modular invariant. The most well-known example is the original construction for the Moonshine Module $V^{\natural}$ as a $\mathbb{Z}_{2}$ orbifold of the Leech lattice VOA [FLM].

\subsection{Permutation Orbifolds}

Let $V^{\otimes n}=V \otimes V \otimes \ldots V$ denote the $n^{\text {th }}$ tensor product VOA with partition function $Z_{V^{\otimes n}}(\tau)=Z(\tau)^{n}$ and central charge $24 n$. The symmetric group $S_{n}$ naturally acts on $V^{\otimes n}$ as an automorphism group. For each $\beta \in S_{n}$ there is a unique $\beta$-twisted $V^{\otimes n}$ module $M_{\beta}$ which can be explicitly constructed from the original HVOA $V$ [DMVV], [BDM], [Ba]. Furthermore, we may explicitly compute the permutation orbifold partition function $Z^{S_{n}-\text { orb }}(\tau)$.

We illustrate this in the first non-trivial case for $V \otimes V$. Then $S_{2}=\langle\sigma\rangle$ for 2-cycle $\sigma$ where $\sigma: u \otimes v \rightarrow v \otimes u$ for all $u \otimes v \in V \otimes V$. We thus find

$$
Z_{V \otimes V}((\sigma, 1), \tau)=Z(2 \tau) .
$$

The $\sigma$-twisted module $M_{\sigma}$ has partition function

$$
Z_{V \otimes V}((1, \sigma), \tau)=\operatorname{Tr}_{M_{\sigma}}\left(q^{L(0)-1}\right)=Z\left(\frac{\tau}{2}\right),
$$

with

$$
Z_{V \otimes V}((\sigma, \sigma), \tau)=Z\left(\frac{\tau+1}{2}\right),
$$


following (24). Thus we obtain

$$
Z^{S_{2}-\mathrm{orb}}(\tau)=\frac{1}{2} Z(\tau)^{2}+T(2) Z(\tau)
$$

for Hecke operator $T(2)$ of (4) for weight zero.

In general, for $\beta \in S_{n}$, consider the cycle decomposition

$$
\beta=\sigma_{1}^{m_{1}} \sigma_{2}^{m_{2}} \ldots \sigma_{n}^{m_{n}}
$$

where $\sigma_{k}$ denotes a $k$-cycle. The conjugacy classes of $S_{n}$ are enumerated by the set of partitions of $n=\sum_{1 \leq k \leq n} k m_{k}$. The centralizer is then

$$
C(\beta)=S_{m_{1}} \times\left(S_{m_{2}} \rtimes C_{2}^{m_{2}}\right) \times \ldots \times\left(S_{m_{n}} \rtimes C_{n}^{m_{n}}\right),
$$

of order $\prod_{1 \leq k \leq n} k^{m_{k}} m_{k}$ ! with cyclic group $C_{k}=\left\langle\sigma_{k}\right\rangle$ and $S_{m_{k}}$ the permutation group on the $m_{k}$ cycles $\sigma_{k}$. We may construct $M_{\beta}=\otimes_{k} M_{\sigma_{k}}^{\otimes m_{k}}$ which has partition function [BDM]

$$
Z((1, \beta), \tau)=\operatorname{Tr}_{M_{\beta}}\left(q^{L(0)-1}\right)=\prod_{1 \leq k \leq n} Z\left(\frac{\tau}{k}\right)^{m_{k}}
$$

One eventually finds that the $S_{n}$ permutation orbifold partition function is DMVV

$$
Z^{S_{n}-\text { orb }}(\tau)=\sum_{[\beta] \in S_{n}} \frac{1}{|C(\beta)|} \sum_{\alpha \in C(\beta)} Z((\alpha, \beta), \tau)=\sum_{\substack{m_{1}, \ldots m_{n} \\ \sum k m_{k}=n}} \prod_{1 \leq k \leq n} \frac{1}{m_{k} !}(T(k) Z(\tau))^{m_{k}}
$$

for the classical Hecke operator $T(k)$ of (4).

It is natural to define a permutation orbifold generating function by

$$
Z^{\text {perm }}(p, q)=1+\sum_{n \geq 1} p^{n} Z^{S_{n}-\mathrm{orb}}(\tau)
$$

for a formal parameter $p$. Thus we obtain [op.cit.]

$$
Z^{\text {perm }}(p, q)=\exp \left(\sum_{n \geq 1} p^{n} T(n) Z(\tau)\right)=\prod_{r \geq 1, s \in \mathbb{Z}} \frac{1}{\left(1-p^{r} q^{s}\right)^{a(r s)}}
$$

where $Z(\tau)=\sum_{k \geq-1} a(k) q^{k}$. This is clearly of the form of the inverse of the LHS of denominator formula (13). Thus such expressions canonically arise in the context of permutation orbifolds for $C=24$ HVOAs. 
Specializing to the case of the Moonshine module $V^{\natural}$ where $Z(\tau)=J(\tau)$ we obtain

$$
\begin{gathered}
Z_{V^{\natural}}^{\text {perm }}(p, q)=\exp \left(\sum_{n \geq 1} p^{n} T(n) J(\tau)\right)=\frac{1}{p J(p)-p J(q)} \\
=1+p J(q)+\left(J(q)^{2}-c(1)\right) p^{2}+\left(J(q)^{3}-2 J(q) c(1)-c(2)\right) p^{3}+ \\
\left(J(q)^{4}-3 c(1) J(q)^{2}-2 J(q) c(2)-c(3)+c(1)^{2}\right) p^{4}+\ldots
\end{gathered}
$$

This formula and the infinite product formula of (32) very strongly suggest that $Z_{V^{\natural}}^{\text {perm }}(p, q)$ is the partition function for a doubly graded symmetric bosonic module with Monster characters which is, algebraically speaking, the inverse of the alternating homological structure constructed by Borcherds [Bo2]. Furthermore, infinite product formulas such as that of (32) have been given the interesting interpretation as a "second quantized" string partition function in the physics literature [DMVV]. A rigorous VOA construction for such a structure would be of obvious interest.

\section{$5 \quad$ Finite Group and Permutation Orbifolds}

Let us now consider orbifolding $V^{\otimes n}$ with respect to $G \times S_{n}$ where $G$ acts diagonally on $V^{\otimes n}$ and $S_{n}$ is the permutation group for $V^{\otimes n}$. We consider again a $C=24$ holomorphic VOA, with modular invariant partition function and where (24) is holds. We may construct unique twisted sectors for each $(h, \beta) \in G \times S_{n}[\mathrm{BDM}]$ to find [T4]

$$
Z^{S_{n}-\mathrm{orb}}((g, h), \tau)=\sum_{\substack{m_{1}, \ldots m_{n} \\ \sum k m_{k}=n}} \prod_{1 \leq k \leq n} \frac{1}{m_{k} !}(T(k) Z((g, h), \tau))^{m_{k}},
$$

where here $T(k)$ is the twisted Hecke Operator of (15). Then (18) implies

$$
\left.(T(n) Z((g, h), \tau))\right|_{0} \gamma=T(n) Z((g, h), \tau) .
$$

We may define a permutation orbifold generating function generalizing (31) as follows:

$$
\begin{aligned}
Z^{\text {perm }}((g, h), p, q) & =\sum_{n \geq 1} p^{\frac{n}{m}} Z^{S_{n}-\text { orb }}((g, h), \tau) \\
& =\exp \left(\sum_{n \geq 1} p^{\frac{n}{m}} T(n) Z((g, h), \tau)\right) .
\end{aligned}
$$

where $h$ is of order $m$ and using (33). For $g=1$, this expression reduces to an infinite product formula generalizing (32) to find

$$
Z^{\text {perm }}((1, h), p, q)=\prod_{r \geq 1, s \in \mathbb{Z}}\left(1-p^{\frac{r}{m}} q^{\frac{s}{m}}\right)^{-a\left(\left(1, h^{r}\right), \frac{r s}{m}\right)},
$$


where $\left.Z\left(\left(1, h^{r}\right), \tau\right)\right)=\sum_{k \in \mathbb{Z}} a\left(\left(1, h^{r}\right), \frac{k}{m}\right) q^{\frac{k}{m}}$.

\section{Monstrous and Generalized Moonshine - the Genus Zero Property}

We now consider the FLM Moonshine Module VOA $V^{\natural}$ [FLM] and its relationship to Moonshine. The original Monstrous Moonshine paper of Conway and Norton described evidence for an unexpected relationship between properties of the Monster finite group and the theory of modular forms [CN]. Many of these relationships are now understood to be generic to orbifold constructions in conformal field theory/VOA theory e.g. [FLM], [T1], [T2], [DLM]. However, the special feature that sets the Moonshine Module $V^{\natural}$ apart from other VOAs is the Genus Zero Property [CN]. This states that for each $g \in \mathbb{M}$, the McKay-Thompson series $T_{g}(\tau)$ of (20) is a hauptmodul for some genus zero modular group $\Gamma_{g}$. Thus for $g$ of prime order $o(g)=p$, one finds (excluding one class of order 3) that either $\Gamma_{g}=\Gamma_{0}(p)$ with $g=p-($ in the notation of [CN]) or else with $g=p+$ with $\Gamma_{g}=\Gamma_{0}(p)+=\left\langle\Gamma_{0}(p), W_{p}\right\rangle$ where $\Gamma_{0}(p)=\left\{\left(\begin{array}{ll}a & b \\ c & d\end{array}\right) \mid c=0 \bmod p\right\}$ and $W_{p}: \tau \rightarrow-1 / p \tau$ is a Fricke involution. In general, we say that $g \in \mathbb{M}$ is Fricke if $T_{g}$ is invariant under a Fricke involution $W_{N}: \tau \rightarrow-1 / N \tau$ where $N=k o(g)$ and $k \mid 24$ and is otherwise non-Fricke. $k=1$ in the global phase anomaly free cases where (24) holds.

The distinction between Fricke and non-Fricke classes is particularly important in the orbifold interpretation of Moonshine [T1]. There is very significant evidence for the general conjecture that the genus zero property for a McKay-Thompson series is equivalent to the statement that for any global phase anomaly free element $g$, orbifolding $V^{\natural}$ with respect to $\langle g\rangle$ for $g$ Fricke results in $V^{\natural}$ again whereas orbifolding $V^{\natural}$ with respect to $\langle g\rangle$ for $g$ non-Fricke results in the Leech lattice VOA [T2].

Generalized Moonshine refers to the still generally unproven conjecture of Norton [N2] that for each commuting pair $g, h \in \mathbb{M}$, then $Z_{V^{\natural}}((g, h), \tau)$ is either a hauptmodul for a genus zero modular group or is a constant. It is easy to show using (23) that (1) $Z_{V^{\natural}}((g, h), \tau)$ is constant iff $g^{c} h^{d}$ is nonFricke for all $(c, d)=1$ [N2], [T3] and (2) if $g, h \in\langle k\rangle$ for some $k \in \mathbb{M}$ then $Z_{V^{\natural}}((g, h), \tau)$ is a hauptmodul (since it can then be modular transformed to a McKay-Thompson series [T3], [IT1], [DLM]). In the remaining "nontrivial" cases, we may use (23) again to transform $Z_{V^{\natural}}((g, h), \tau)$ to a trace over a Fricke twisted module so that the genus zero property reduces to an analysis of $h$ Fricke cases alone. The case of $h=2+$, with centralizer 2.B for the Baby Monster $B$, has now been proved by Hoehn $[\mathrm{H}]$. The relationship 
between orbifoldings of the Moonshine module and the genus zero property of Generalized Moonshine for $h=p+$ is discussed at length in [T3], [IT1], [IT2, [I].

The approach taken in Borcherds' proof of the Monstrous Moonshine genus zero property is to firstly prove a twisted denominator identity generalizing (13) [N1], Bo2]

$$
\exp \left(-\sum_{n \geq 1} p^{n} T(n) T_{g}(\tau)\right)=p\left(T_{g}(p)-T_{g}(q)\right)
$$

This is the defining formula for completely replicable functions [N1], [FMN] from which it follows that the leading coefficients of $T_{g^{i}}(\tau)$ for $i=1,2, \ldots$ determine $T_{g}(\tau)$. Koike showed that the list of hauptmoduln appearing in the Moonshine Conjectures are completely replicable [Ko]. Based on this result and an analysis of the leading coefficients (using the explicit form for $T_{g}(\tau)$ found by FLM for 2- centralizers in the Monster [FLM]) Borcherds then demonstrated that indeed $T_{g}(\tau)$ obeys the genus zero property. This part of the proof was improved upon in [CG where meromorphicity, modularity and the genus zero property are shown to generally follow from the infinitely many replication formulas that follow from (1) and (37), namely

$$
T(n) T_{g}(q)=\frac{1}{n} F_{n}\left(T_{g}(q)\right),
$$

where $F_{n}$ is the Faber polynomial for $T_{g}(q)$.

However, as already noted, $T_{g}(q)$ is known to be meromorphic on $\mathbb{H}$ from [DLM]. Furthermore, by combining (38) with the general modular transformation property (23) (or (24) in the absence of global anomalies) one can also expect to obtain the genus zero property for $T_{g}$ in a more direct fashion along the lines of the methods described in refs. [T1], [T2].

On the other hand, in the permutation orbifold construction based on $V^{\natural}$, (37) reads

$$
\begin{aligned}
Z_{V^{\natural}}^{\text {perm }}((g, 1), p, q) & =\exp \left(\sum_{n \geq 1} p^{n} T(n) Z_{V^{\natural}}((g, 1), q)\right) \\
& =\frac{1}{p\left(Z_{V^{\natural}}((g, 1), p)-Z_{V^{\natural}}((g, 1), q)\right)} .
\end{aligned}
$$

Recall our previous remarks concerning the reduction of Generalized Moonshine to Fricke classes. Consider $h$ a global phase anomaly-free Fricke element of order $o(h)=m$ so that $Z_{V^{\natural}}\left((1, h), q^{m}\right)=Z_{V^{\natural}}((h, 1), q)$. It follows that

$$
\exp \left(\sum_{n \geq 1} p^{n} T(n) Z\left((1, h), q^{m}\right)\right)=\frac{1}{p\left(Z_{V^{\natural}}\left((1, h), p^{m}\right)-Z_{V^{\natural}}\left((1, h), q^{m}\right)\right)} .
$$


Hence from (36) we find for such Fricke $h$ that

$$
Z_{V^{\natural}}^{\text {perm }}((1, h), p, q)=\frac{1}{p^{\frac{1}{m}}\left(Z_{V^{\natural}}((1, h), p)-Z_{V^{\natural}}((1, h), q)\right)} .
$$

This together with the general result (36) again suggests the existence of a symmetric bosonic construction forming a doubly-graded module for the centralizer of $C(h)$ for each such Fricke element $h$. It is thus natural to conjecture that for all order $m$ global phase anomaly-free Fricke elements $h$ the following holds

$$
\begin{aligned}
Z_{V^{\natural}}^{\text {perm }}((g, h), p, q) & =\exp \left(\sum_{n \geq 1} p^{\frac{n}{m}} T(n) Z_{V^{\natural}}((g, h), q)\right) \\
& =\frac{1}{p^{\frac{1}{m}}\left(Z_{V^{\natural}}((g, h), p)-Z_{V^{\natural}}((g, h), q)\right)} .
\end{aligned}
$$

From (1), this is equivalent to the following Generalized Moonshine replication formula for global phase anomaly-free Fricke elements $h$

$$
T(n) Z_{V^{\natural}}((g, h), q)=\frac{1}{n} F_{n}\left(Z_{V^{\natural}}((g, h), q)\right),
$$

where $F_{n}$ is the Faber polynomial for $\left.Z_{V^{\natural}}\left((g, h), q^{m}\right)\right)$. The equivalence of this replication formula to the genus zero property for Generalized Moonshine would therefore require a suitable generalization of the various Monstrous Moonshine arguments.

We conclude with the example of $n=2$. Then (41) implies

$$
\begin{aligned}
& Z_{V^{\natural}}\left(\left(g^{2}, h\right), 2 \tau\right)+Z_{V^{\natural}}\left(\left(g, h^{2}\right), \frac{\tau}{2}\right)+Z_{V^{\natural}}\left(\left(g h, h^{2}\right), \frac{\tau+1}{2}\right) \\
= & Z_{V^{\natural}}((g, h), \tau)^{2}-2 a\left((g, h), \frac{1}{m}\right),
\end{aligned}
$$

where $Z_{V^{\natural}}((g, h), \tau)=q^{-\frac{1}{m}}+0+a\left((g, h), \frac{1}{m}\right) q^{\frac{1}{m}}+\ldots$ which corresponds to an example quoted in ref. [N2]. In particular, for $h=2+$ and $g$ of order 2 then using Fricke invariance we find

$$
T_{h}(\tau)+T_{g}\left(\frac{\tau}{2}\right)+T_{g h}\left(\frac{\tau+1}{2}\right)=Z_{V^{\natural}}((g, h), \tau)^{2}-2 a\left((g, h), \frac{1}{2}\right),
$$

which can be easily verified in each case.

\section{References}

[Ba] Bantay, P.: Permutation orbifolds, Nucl.Phys. B633, 365-378 (2002). 
[BDM] Barron, K., Dong, C. and Mason, G.: Twisted sectors for tensor product vertex operator algebras associated to permutation orbifolds. Commun.Math.Phys. 227, 349-384 (2002).

[Bo1] Borcherds, R.: Vertex algebras, Kac-Moody algebras and the Monster. Proc.Natl.Acad.Sci.U.S.A. 83, 3068-3071 (1986).

[Bo2] Borcherds, R.: Monstrous moonshine and monstrous Lie superalgebras. Invent.Math. 109, 405-444 (1992).

[CG] Cummins, C.J. and Gannon, T.: Modular equations and the genus zero property of moonshine functions, Invent.Math. 129, 413-443 (1997).

[Cu] Curtiss, J. H.: Faber Polynomials and the Faber Series, Am. Math. Mon. 78 577-596 (1971).

[CN] Conway, J.H. and Norton, S.P.: Monstrous moonshine. Bull. London Math. Soc. 11, 308-339 (1979).

[DLM] Dong, C., Li, H. and Mason, G.: Modular-invariance of trace functions in orbifold theory and generalized moonshine. Commun.Math.Phys. 214, 1-56 (2000).

[DM] Dong, C. and Mason, G., Holomorphic vertex operator algebras of small central charge, Pac.J.Math., 2004, 213 253-266.

[DMVV] Dijkgraaf, R., Moore, G., Verlinde, E. and Verlinde, H.: Elliptic genera of symmetric products and the second quantized string, Commun.Math.Phys. 185, 197-209 (1997).

[F] Ferenbaugh, C.: Lattices and generalized Hecke operators, in Groups, difference sets and the Monster, Walter de Gruyter, (1996).

[FMN] Ford, D., McKay, J. and Norton, S.P.: More on replicable functions. Commun.Algebra 22, 5175-5193 (1994).

[FLM] Frenkel, I., Lepowsky, J. and Meurman, A.: Vertex operator algebras and the Monster. New York:Academic Press, 1988.

[H] Hoehn, G.: Generalized moonshine for the baby monster, Talk presented at this conference.

[I] Ivanov, R.I.: Generalised Moonshine from Abelian Orbifolds of the Moonshine Module. Ph.D. Thesis, National University of Ireland, Galway, 2002. 
[IT1] Ivanov, R.I. and Tuite, M.P.: Rational generalised moonshine and abelian orbifolds. Nucl.Phys. B635 (2002), 435-472.

[IT2] Ivanov, R.I. and Tuite, M.P.: Some irrational generalised moonshine from orbifolds. Nucl.Phys. B635 (2002), 473-491.

[Ka] Kac, V.: Vertex Operator Algebras for Beginners. University Lecture Series, Vol. 10, Boston:AMS 1998.

[Ko] Koike, M.: On replication formula and Hecke operators, Nagoya University preprint.

[MN] Matsuo, A. and Nagatomo, K.: Axioms for a vertex algebra and the locality of quantum fields, Math.Soc.Japan Memoirs. 4, (1999).

[MTZ] Mason, G., Tuite, M.P. and Zuevsky, A.: Torus $n$-Point Functions for $\mathbb{R}$-graded Vertex Operator Superalgebras and Continuous Fermion Orbifolds, Commun.Math.Phys. 283, 305-342 (2008).

[N1] Norton, S. P.: More on moonshine, in Computational Group Theory, Academic Press, 185-193 (1984).

[N2] Norton, S.P.: Generalised moonshine. Proc.Symp.PureMath. 47, 208-210 (1987).

[Sch] Schellekens, A.N.: Meromorphic c $=24$ conformal field theories, Commun.Math.Phys. 153, 159-186 (1993).

[Se] Serre, J-P.: A course in arithmetic, Springer-Verlag (Berlin 1978).

[T1] Tuite, M. P.: Monstrous moonshine from orbifolds. Commun.Math.Phys. 146 277-309 (1992).

[T2] Tuite, M. P.: On the relationship between monstrous moonshine and the uniqueness of the moonshine module. Commun.Math.Phys. 166, 495-532 (1995).

[T3] Tuite, M. P.: Generalised moonshine and abelian orbifold constructions. Contemp.Math. 193, 353-368 (1996).

[T4] Tuite, M. P.: To appear.

[Va] Vafa, C.: Modular invariance and discrete torsion on orbifolds. Nucl.Phys. B273, 592-606 (1986).

[Z] Zhu, Y.: Modular invariance of characters of vertex operator algebras. J.Amer.Math.Soc. 9, 237-302 (1996). 